\title{
PRODUÇÃo DE MICROBOLHAS PARA A FLOTAÇÃO POR AR INDUZIDO
}

\author{
R. C. S. HENAUTH ${ }^{1,3}$, R. S. VASCONCELOS ${ }^{1,3}$, A. E. MOURA ${ }^{2,3}$, L. A. SARUBBO ${ }^{1,3}$ e V. A. \\ SANTOS $^{1,3}$ \\ ${ }^{1}$ Universidade Católica de Pernambuco, Centro de Ciência e Tecnologia; \\ ${ }^{2}$ Universidade Federal de Pernambuco, Departamento de Energia Nuclear; \\ ${ }^{3}$ Centro de Gestão de Tecnologia e Inovação (CGTI) \\ E-mail para contato: rosangelahenauth@yahoo.com.br
}

\begin{abstract}
RESUMO - Com a finalidade de analisar as condições de produção de microbolhas foi aplicado um delineamento composto central rotacional (DCCR), do tipo $2^{2}$, para definir parâmetros operacionais em uma instalação piloto de flotação por ar induzido (FAI). Para variáveis independentes foram utilizadas a pressão manométrica da linha de descarga da bomba de microbolhas $\left(X_{l}\right)$ e a razão entre as vazões de água e de ar $\left(X_{2}\right)$. Como variável resposta foi utilizada a eficiência de produção de microbolhas, quantificada pelo cálculo da eficiência de separação água/óleo ( $\eta)$. Observou-se que $\eta$ passou por condições otimizadas para valores de $X_{1}$ e de $X_{2}$ em torno de 1000 e 3,8 , respectivamente. $\mathrm{O}$ valor de $X_{1}$ ficou limitado pelas condições de cavitação criadas devido à injeção de ar na linha de aspiração da bomba. $\mathrm{O}$ valor de $X_{2}$ confirmou níveis esperados de pressão. A metodologia proposta para a produção de microbolhas mostrou-se vantajosa em comparação com processos convencionais de saturação da água a ser tratada com ar comprimido, pela redução de equipamentos e custos com energia.
\end{abstract}

\section{INTRODUÇÃO}

Na indústria, em geral, há uma demanda muito grande por água de boa qualidade e grande parte do referido volume será eliminado para corpos receptores com uma alta carga de matéria orgânica e sólidos, motivo pelo qual águas residuárias geradas em todos os processos industriais devem passar por um tratamento específico. Embora seja, teoricamente, possível tratar o efluente para qualquer padrão requerido, existem fatores limitantes como os custos de capital, orçamento operacional e espaço físico. As etapas primárias de tratamento podem ser definidas como responsáveis pela remoção da maior parte da matéria poluidora e preparação do efluente para a etapa posterior (SCHOENHALS, 2006). 


\section{9 a 22 de outubro de 2014 \\ Florianópolis/SC}

Dentre os vários processos de tratamento de efluentes, podem-se destacar os tratamentos físicos, que são caracterizados por métodos de separação de fases: sedimentação, decantação, filtração, centrifugação ou flotação dos resíduos (CRESPILHO et al., 2004). Com o rápido desenvolvimento da tecnologia, a flotação tem sido amplamente utilizada no tratamento de efluentes industriais, sendo um processo simples de se implementar, fácil de administrar, de baixo custo, podendo, ainda ser utilizado numa grande variedade de processos, ou seja, a separação através da flotação é um processo barato, porque não há grandes operações consumidoras de energia, em comparação com algo como destilação ou evaporação (JORDAN, 2013).

Na flotação por ar induzido convencional (FAI), um sistema constituído de um compressor de ar gera um fluxo contínuo de microbolhas que se inicia na parte mais inferior do recipiente de tratamento, em direção à superfície do líquido. Ao entrarem em contato com estas microbolhas, as impurezas presentes no efluente aderem à superfície das mesmas, formando assim um agregado bolhas-partícula (MAIA et al., 2012). Os agregados têm densidade menor que a fase líquida e ascendem à superfície arrastando as partículas seletivamente aderidas e promovendo a separação da mistura. Esse fenômeno se deve à tensão superficial do meio de dispersão e ao ângulo de contato formado entre as bolhas e as partículas (MASSI et al., 2008). O processo de flotação por gás dissolvido (FGD) refere-se à flotação realizada na presença de gás dissolvido, como fonte geradora de bolhas. Na prática, qualquer gás pode ser usado, mas devido ao custo, o ar é comumente empregado na maioria das aplicações industriais. Assim, a flotação por gás dissolvido é denominada mais especificamente como flotação por ar dissolvido (FAD) (RODRIGUES, 2004). A diferença fundamental entre os sistemas FAI e FAD é o mecanismo pelo qual as bolhas de ar são introduzidas no líquido (BENNETT, 1988).

A FAI com a utilização de microbolhas $(<100 \mu \mathrm{m})$ é uma técnica bem conhecida para a remoção de sólidos, fibras, micro-organismos e outras impurezas na água e no tratamento de águas residuárias (RUBIO et al., 2002; LI e TSUGE, 2006; CARISSIMI et al., 2007; RODRIGUES e RUBIO, 2007; ENGLERT et al., 2009). Apesar de alguns autores relatarem benefícios em relação a minerais serem recuperados pela FAI, a sua aplicação para a separação desses minérios é bastante pobre, principalmente devido à baixa capacidade de carga das bolhas finas, com bolhas maiores (limitando os tamanhos de partículas que podem flutuar) (PÉREZGARIBAY et al., 2012).

Dentre as metodologias de avaliação da eficiência de um processo, a exemplo da produção 
de microbolhas, está à ferramenta estatística descrita domo delineamento composto central rotacional (DCCR). O DCCR consiste, basicamente, na seleção de um número fixo de níveis para cada um dos fatores ou variáveis e na execução de experimentos com todas as combinações possíveis (CAPELLARI, 2010). Consta de uma parte fatorial, uma parte axial e pontos centrais (CONAGIN, 1982). Esse tipo de delineamento foi desenvolvido por Box e Wilson (1951), visando inicialmente a sua utilização em pesquisas da indústria, bem como na química. Esses pesquisadores afirmam que o delineamento composto central rotacional da superfície de resposta é o método de análise de resultados que proporciona um número maior de informações.

Neste trabalho desenvolveu-se uma técnica do tipo FAI sem a utilização de compressor. A vazão necessária de ar foi admitida no processo à pressão atmosférica. Dessa forma, utilizou-se um DCCR para estudar as influências de variáveis controladas e de suas possíveis interações no processo de produção dessas microbolhas, na eficiência de separação água-óleo.

\section{METODOLOGIA}

Para avaliação da eficiência de produção das microbolhas, aplicou-se um DCCR. As variáveis estudadas foram pressão manométrica $\left(X_{1}\right)$ e a razão entre as vazões de ar/água $\left(X_{2}\right)$. Na Tabela 1 são apresentadas as faixas de valores reais codificados utilizados para os ensaios.

Tabela 1 - Faixas de valores reais e codificados utilizados no DCCR

\begin{tabular}{cccccc}
\hline & Nível & & \\
\hline Fatores & $-1,41$ & $-1,0$ & 0 & $+1,0$ & $+1,41$ \\
$\boldsymbol{X}_{\boldsymbol{I}}(\mathbf{b a r})$ & 1,186 & 1,6 & 2,6 & 3,6 & 4,014 \\
$\left.\boldsymbol{X}_{\mathbf{2}} \mathbf{( 1 \mathbf { 1 0 }}^{\mathbf{3}}\right)$ & 0,234 & 0,4 & 0,8 & 1,2 & 1,366 \\
\hline
\end{tabular}

O DCCR foi do tipo $2^{2}$, totalizando 12 experimentos, sendo os mesmos executados em sequência aleatória conforme em uma matriz de planejamento. As distâncias dos pontos axiais foram $\pm 1,41$, sendo os dados tratados com o auxílio do software Statistica 10.0 Statsoft ${ }^{\circledR}$. Esse tipo de planejamento permite a obtenção de modelos matemáticos com parâmetros lineares e quadráticos que descrevem o comportamento do processo na faixa estudada. Além disso, são calculados os efeitos principais e de interação das variáveis que influenciam a resposta, bem como os respectivos coeficientes de regressão oriundos do modelo matemático (LIED, 2012). 


\section{RESULTADOS}

Os valores determinados na Tabela 1 geraram os resultados de ensaios preliminares, conforme apresentados na Tabela 2. Os valores máximos de eficiência mostrados pela Tabela 2 encontram-se nos pontos centrais, numa demonstração de uma faixa de valores adequados nos experimentos realizados.

Tabela 2 - Delineamento Composto Central Rotacional (DCCR) com variáveis independentes em valores codificados e reais e variável dependente com valores experimentais

\begin{tabular}{cccccc}
\hline & \multicolumn{2}{c}{$\begin{array}{c}\text { Valores } \\
\text { Codificados }\end{array}$} & \multicolumn{2}{c}{$\begin{array}{c}\text { Valores } \\
\text { Reais }\end{array}$} & $\begin{array}{c}\text { Valores } \\
\text { Experimentais }\end{array}$ \\
\hline $\begin{array}{c}\mathbf{N}^{\mathbf{0}} \mathbf{d e} \\
\text { Ensaios }\end{array}$ & $\mathbf{X}_{\mathbf{1}}$ & $\mathbf{X}_{\mathbf{2}}$ & $\mathbf{X}_{\mathbf{1}}$ & $\mathbf{X}_{\mathbf{2}}$ & $\mathbf{Y}$ \\
$\mathbf{1}$ & -1 & -1 & 1,600000 & 0,400000 & 23 \\
$\mathbf{2}$ & -1 & 1 & 1,600000 & 1,200000 & 70 \\
$\mathbf{3}$ & 1 & -1 & 3,600000 & 0,400000 & 26 \\
$\mathbf{4}$ & 1 & 1 & 3,600000 & 1,200000 & 59 \\
$\mathbf{5}$ & $-1,41421$ & 0 & 1,185786 & 0,800000 & 44 \\
$\mathbf{6}$ & 1,41421 & 0 & 4,014214 & 0,800000 & 25 \\
$\mathbf{7}$ & 0 & $-1,41421$ & 2,600000 & 0,234315 & 15 \\
$\mathbf{8}$ & 0 & 1,41421 & 2,600000 & 1,365685 & 62 \\
$\mathbf{9}$ & 0 & 0 & 2,600000 & 0,800000 & 75 \\
$\mathbf{1 0}$ & 0 & 0 & 2,600000 & 0,800000 & 77 \\
$\mathbf{1 1}$ & 0 & 0 & 2,600000 & 0,800000 & 78 \\
$\mathbf{1 2}$ & 0 & 0 & 2,600000 & 0,800000 & 74 \\
\hline
\end{tabular}

Os resultados da análise de variância (ANOVA), que indica a predição de um modelo de previsão com nível de confiança de $95 \%$, são apresentados na Tabela 3. A correlação entre os dados experimentais e o valor predito pelo modelo foi satisfatório $\left(\mathrm{R}^{2}=0,96823\right)$. Aplicando a distribuição de Fisher (teste F) ao modelo foi possível observar que o mesmo foi significativo e preditivo.

Tabela 3 - Análise de variância (ANOVA)

\begin{tabular}{cccccc}
\hline Fator & SS & df & MS & F & P \\
\hline$(\mathbf{1}) \boldsymbol{X}_{\boldsymbol{I}}(\mathbf{b a r})(\mathbf{L})$ & 151,990 & 1 & 151,990 & 45,5970 & 0,006634 \\
$\boldsymbol{X}_{\boldsymbol{I}}$ (bar) $(\mathbf{Q})$ & 2250,000 & 1 & 2250,000 & 675,0000 & 0,000125 \\
\hline
\end{tabular}


(2) $X_{2}\left(1 \times 10^{3}\right)(\mathrm{L})$

$X_{2}\left(1 \times 10^{3}\right)(Q)$

$\mathrm{X}_{1} \mathrm{X}_{2}$

Falta de ajuste

Erro experimental

Total SS

\begin{tabular}{ccccc}
2681,611 & 1 & 2681,611 & 804,4832 & 0,000096 \\
1795,600 & 1 & 1795,600 & 538,6800 & 0,000175 \\
49,000 & 1 & 49,000 & 14,7000 & 0,031275 \\
195,399 & 3 & 65,133 & 19,5399 & 0,017968 \\
10,000 & 3 & 3,333 & & \\
6464,667 & 11 & & & \\
\hline
\end{tabular}

No diagrama de Pareto, exposto pela Figura 1, os fatores que ultrapassam a linha pontilhada representam as variáveis com efeito significante sobre a variável resposta com $95 \%$ de confiança. A partir desse gráfico observa-se que todas as variáveis são, estatisticamente, significantivas, com destaque para o valor positivo do termo linear da razão entre as vazões ar/água e valores negativos dos demais termos. Isso leva a se observar que os demais termos têm valores críticos, acima dos quais a eficiência de separação água/óleo decresce. Por outro lado, a interação entre os fatores mostrou-se pouco significativa, de modo a se poder variar qualquer um deles para se obter um efeito desejado sem interferência na contribuição do outro fator.

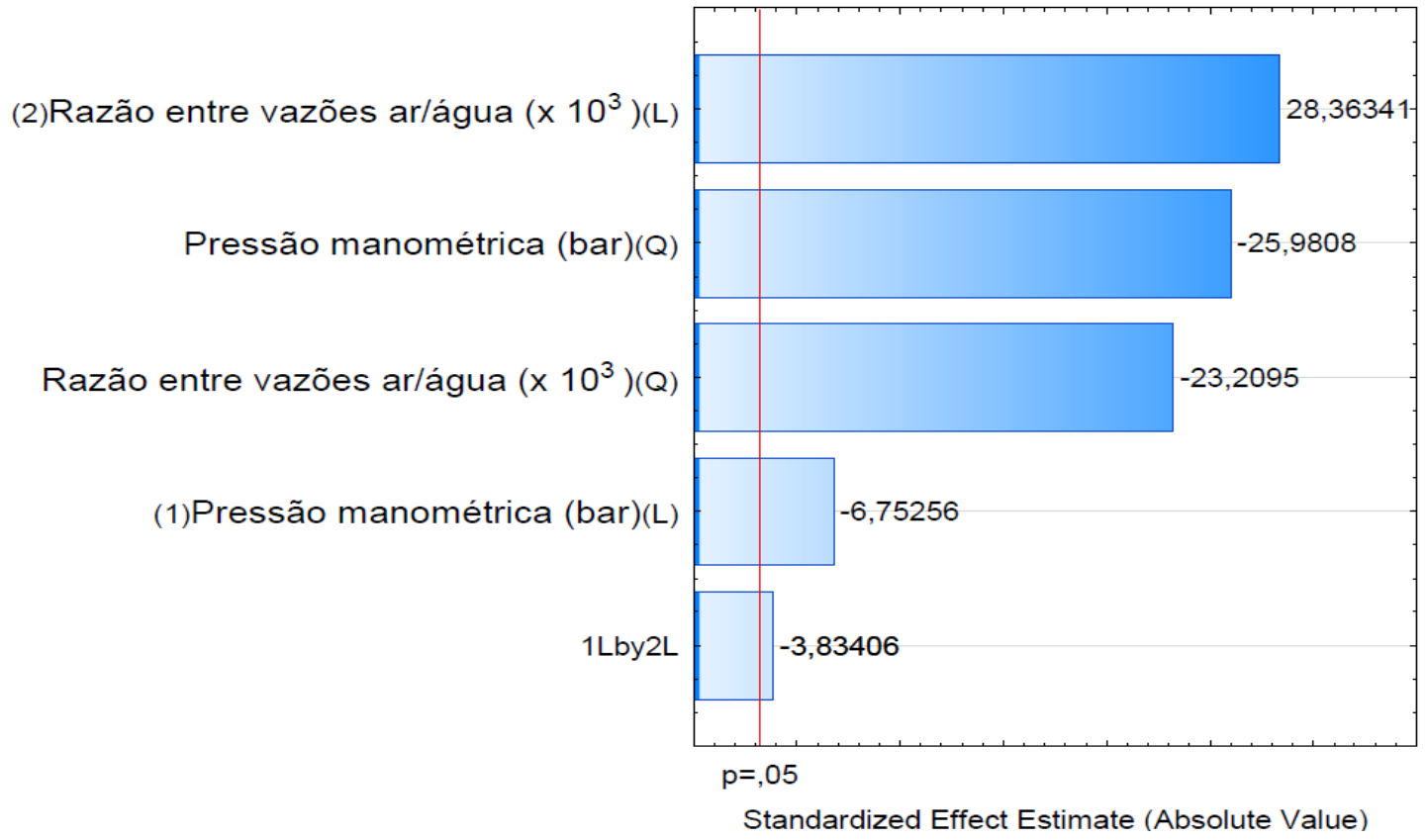

Figura 1 - Diagrama de Pareto.

Os coeficientes dos fatores $\left(X_{1}\right.$ e $\left.X_{2}\right)$ no modelo de previsão foram determinados correlacionando-se os dados experimentais com auxílio da tabela de ANOVA (Tabela 3). O modelo de previsão obtido para a eficiência de produção de microbolhas $(Y)$, recebeu uma 
variância explicada, ou 96,82 \% de explicação para as variações no fenômeno estudado. A Equação 1 apresenta o modelo de previsão obtido:

$$
Y=161,23+100,14 X_{1}-18,75 X_{1}{ }^{2}+236,02 X_{2}-104,68 X_{2}{ }^{2}-8,75 X_{1} X_{2}
$$

Como uma quantidade maior de microbolhas produzida resulta em um número maior de gotas de óleo envolvidas e flotadas, associou-se a eficiência de produção de microbolhas à eficiência de separação água/óleo. A superfície de resposta mostrada na Figura 2, obtida com auxílio da Equação 1 ilustra a dependência da eficiência de produção de microbolhas com a pressão manométrica $\left(X_{1}\right)$ e a razão entre as vazões ar/água $\left(X_{2}\right)$. Valores experimentais desses dois fatores da ordem de 1000 e 3,8, respectivamente originaram o valor máximo para eficiência de produção de microbolhas.

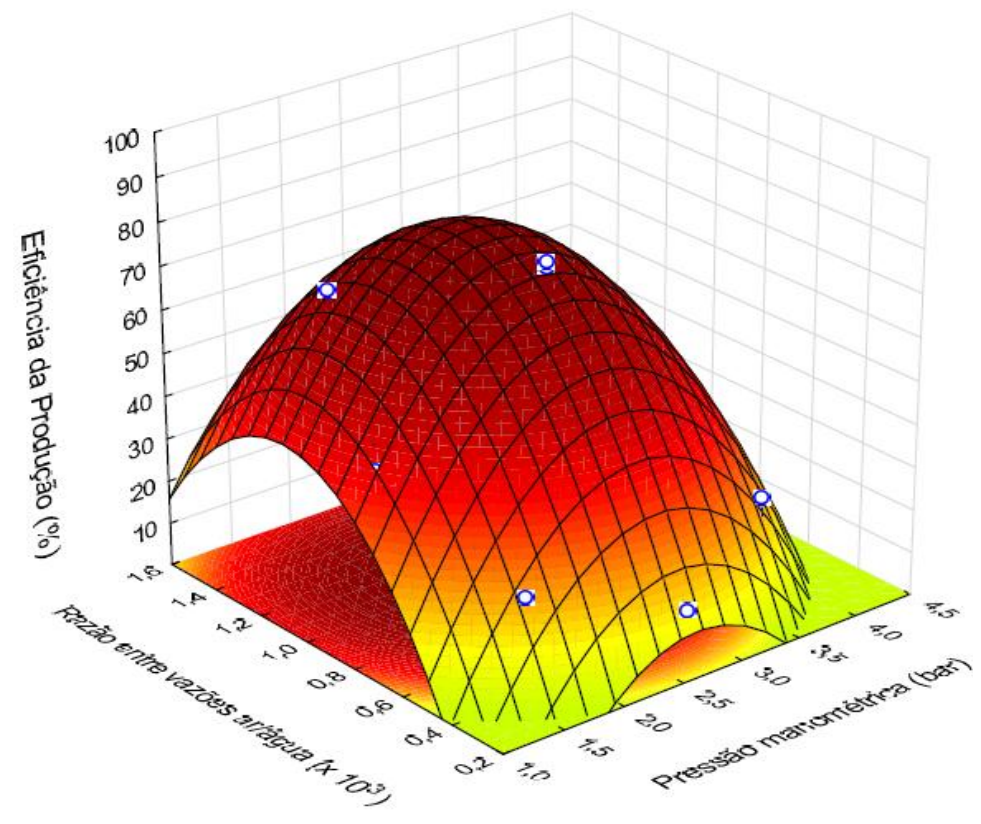

Figura 2 - Superfície de resposta estimada pelo modelo matemático.

\section{CONCLUSÕES}

O desenvolvimento de um sistema para tratamento de águas oleosas utilizando a tecnologia de flotação por ar induzido (FAI), proposta por esse trabalho, permitiu a obtenção de vantagens frente ao processo de produção de microbolhas convencional. A não utilização de equipamentos 
que ocupam consideráveis espaços físicos como compressor e tanque de saturação e a redução no consumo de energia pelo trabalho com ar a pressão ambiente foram vantagens que podem ser utilizadas quando da adoção do FAI para tratamento de águas oleosas.

\section{AGRADECIMENTOS}

À FACEPE, pela concessão da bolsa de Mestrado, ao Centro de Gestão de Tecnologia e Inovação (CGTI), pelo apoio técnico concedido e à UNICAP, pela oportunidade de realização do Mestrado.

\section{REFERENCIAS}

BENNETT, G. F. The Removal of Oil From Wastewater by Flotation: A review. Crit. Rev. Env. Control, v. 18, n. 3, p. 189 - 253, 1988.

BOX, G. E. P.; WILSON, K. B. On The Experimental Attainment of Optimum Conditions. $J$. $R$. Stat. Soc., Ser. B (Statistical Methodology), Manchester, v. 13, n. 1, p.1 - 38, 1951.

CAPELLARI, J. B. Biossíntese de Ácido Láctico por Lactobacillus Amylovorus a Partir de Resíduos Agroindustriais. 2010. 67p. Dissertação (Engenharia de Processos) Universidade da Região de Joinville - UNIVILLE, Joinville, 2010.

CARISSIMI, E.; RUBIO, J.; ROSA, J. J. Flotation in Water And Wastewater Treatment And Reuse: recent trends in Brazil. Int. J. Environ. Pollut., v. 30, n. 2, p. 193 - 208, 2007.

CONAGI, A. Delineamento Composto Central com Duas Estrelas. Pesq. Agropec. Bras. v. 17, n. 9, p. $1261-9.1982$.

CRESPILHO, F. N.; SANTANA, C. G.; REZENDE, M. O. O. Tratamento de efluente de indústria de processamento de coco utilizando eletroflotação. Quím. Nova, v. 27, n. 3, p. 387 - 392, mai./jun. 2004.

ENGLERT, A. H.; RODRIGUES, R. T.; RUBIO, J. Dissolved Air Flotation (DAF) of Fine Quartz Particles Using an Amine as Collector. Int. J. Miner. Process., v. 90, n. 1, p. 27 34, 2009. 
JORDAN, A. T. Liquid Phase Plasma Thecnology Inkjet Separation. 2013. 71p. Dissertação (Mestrado em Engenharia Química e Biomolecular) - Institute of Technology, Georgia, 2013.

LI, P.; TSUGE, H. Water Treatment by Induced Air Flotation Using Microbubbles. J. Chem. Eng. Jpn, v. 39, n. 8, p. 896 - 903, 2006.

LIED, E. B. Tratamento de Efluente Industrial de Fecularia Utilizando Macrófita Aquática Eichhornia crassipes e Coagulante Natural. 2012. 116p. Dissertação (Engenharia Química) - Universidade Estadual do Oeste do Paraná (UNIOESTE), Toledo, Paraná. 2012.

MAIA, A. A.; SILVA, D. A.; LIMA, D. E. S.; DE LIMA, W, J. F. Automação Para Tratamento de Água Por Floculação e Flotação. Contr. \& Autom., 2012.

MASSI, L.; SOUZA, S. R.; LALUCI, C.; JAFELICCI JÚNIOR, M. Fundamentos e Aplicação da Flotação como Técnica de Separação de Misturas. QNEsc, n. 28, p. 20 - 23. 2008.

PÉREZ-GARIBAY, R.; MARTÍNEZ-RAMOS, E.; RUBIO, J. Gas Dispersion Measurements in Microbubble Flotation Systems. Min. Eng., v. 26, n. 15, p. 34 - 40, 2012.

RODRIGUES, R. T. Desenvolvimento da Técnica LTM-Bsizer Para a Caracterização de Bolhas e Avaliação de Parâmetros no Processo de Flotação. 2004. 114p. Tese (Doutorado em Engenharia de Minas, Metalúrgica e de Materiais) - Universidade Federal do Rio Grande do Sul, Porto Alegre, 2004.

RODRIGUES, R. T.; RUBIO, J. DAF - Dissolved Air Flotation: potential applications in the mining and mineral processing industry. Int. J. Miner. Process., v. 82, n. 1, p.1 - 13, 2007.

RUBIO, J.; SOUZA, M. L.; SMITH, R. W. Overview of Flotation as a Wastewater Treatment Techn. Miner. Eng., v. 15, p. 139-155, 2002.

SCHOENHALS, M. Avaliação da Eficiência do Processo de Flotação Aplicado ao Tratamento de Efluentes de Abatedouro Avícola. 2006. 87p. Dissertação (Mestrado em Engenharia Química) - Universidade Federal de Santa Catarina, Florianópolis, 2006. 